\title{
APPROXIMATION OF A LINEAR DYNAMIC PROCESS MODEL USING THE FREQUENCY APPROACH AND A NON-QUADRATIC MEASURE OF THE MODEL ERROR
}

\author{
KRZYSZTOF B. JANISZOWSKI \\ Institute of Automatic Control and Robotics \\ Warsaw University of Technology, ul. A. Boboli 8, 02-525 Warsaw, Poland \\ e-mail:kjanisz@mchtr.pw.edu.pl
}

\begin{abstract}
The paper presents a novel approach to approximation of a linear transfer function model, based on dynamic properties represented by a frequency response, e.g., determined as a result of discrete-time identification. The approximation is derived for minimization of a non-quadratic performance index. This index can be determined as an exponent or absolute norm of an error. Two algorithms for determination of the approximation coefficients are considered, a batch processing one and a recursive scheme, based on the well-known on-line identification algorithm. The proposed approach is not sensitive to local outliers present in the original frequency response. Application of the approach and its features are presented on examples of two simple dynamic systems.
\end{abstract}

Keywords: approximation method, frequency domain, non-quadratic criterion, recursive algorithm.

\section{Introduction}

Investigation of process dynamics may result in different representations. Commonly used identification schemes (Ljung and Söderström, 1987) result in determination of linear parametric models, which have compact form of some transfer function, sometimes of a quite high order. Before application of this model in controller design, it is helpful to get a simplified, lower-order transfer function in a continuous time or a discrete time domain. In the case of determination of a fuzzy parameterized TSK model, a simplified representation is very advantageous, too. In the case of, e.g., determination of a model composed of a static actuator part and an inertial representation of a dynamic part, the simplified model is valuable, too. A common feature for all the mentioned problems is the possibility of determination of a frequency characteristic for the investigated model. Then the underlying computational problem is presented as determination of a linear transfer function model, derived from the known frequency characteristic.

In electrical-engineering applications, the fitting of an approximation in frequency domain is often considered during the modelling of parts of energy transfer components (Deschrijver et al., 2007; Gustavsen and Mo, 2007; Gustavsen, 2004; Lima et al., 2005; Mohan et al., 2004; Unbehauen and Rao, 1997), hence this problem is deeply investigated in terms of proper soft modelling (Deschrijver et al., 2007; Grivet-Talocia et al., 2005; Gustavsen, 2006; 2004) and even building some hardware models (Mohan et al., 2004). In the area of control techniques, various ways are used (Kowalczuk and Kozłowski, 2011; Pintelon and Schoukens, 2004; Sreeram and Agatokhlis, 1991; Unbehauen and Rao, 1997; Varricchio et al., 2004; Wahlberg and Mäkilä, 1996), but usually they are focused on weighted approximation with the least-squares measure of approximation errors.

Various approaches are used to determine these approximations (Ljung and Söderström, 1987). The problem of fitting a reasonable model for some frequency characteristic was formulated long ago (Levy, 1959) and investigated by many authors in classic transfer function form (Ljung and Söderström, 1987; Pintelon and Schoukens, 2004) or other representations (Wahlberg and Mäkilä, 1996). Various techniques are suggested, and among them most interesting are methods based on the basic approach of vector fitting and its ortho normal version (Deschrijver et al., 2010; 2011; 2007; Grivet-Talocia et al., 2005; Gustavsen and Mo, 2007; Gustavsen, 2006; 2004; Gustavsen and Semlyen, 1999). These approaches are usually focused on determination 
of poles of the final transfer function and can be very precise. Based on measured and recorded frequency characteristics, the resulting models can fit to the data with negligible errors; however, some problems with outliers points have been observed and the $l_{1}$ norm was used in the fitting algorithm (Deschrijver et al., 2010; 2011). Similar problems are observed during the fitting of continuous time models (Kowalczuk and Kozłowski, 2011), too.

The approach presented in the above-mentioned works is usually based on the assumption of the linear behaviour of processes considered and a proper choice of poles for model fitting to the estimated frequency plots. In identification of processes with dynamics reflecting some non-linear phenomena, e.g., saturation or hysteresis, the process behaviour depends on working conditions and sometimes is modelled by neural nets. The corresponding frequency plots, developed for these processes, can represent other transients than the usually investigated development of the model with the vector fitting approach. The vector fitting algorithms discussed above are focused on proper selection of transfer function poles and can be less efficient in the mentioned cases. The problem considered in the paper is focused on fitting a frequency response to the data that can have some outliers, far from any rational frequency response, and therefore the fitting algorithm can be defined in way different from usual least-squares algorithms.

An approximation with a non-quadratic performance index has been considered in various contributions (Fiodorov, 1994; Janiszowski, 1998; Kozłowski, 2003; Kowalczuk and Kozłowski, 2011), and some positive aspects of this approach have been pointed out. However, numerical problems with the convergence of the resulting algorithms have been mentioned, too. Recent applications (Deschrijver et al., 2010; 2011) confirm an important feature of the non-quadratic performance index that the resulting models reflect low sensitivity to local distortions of the original frequency characteristic.

The problem of fitting a model to a given frequency characteristic, defined by a set of values $F R=$ $\left.\left\{c_{1}, \ldots, c_{N}\right\}, c_{k}=F R\left(\omega_{k}\right) \in \mathbb{C}\right)$, is usually defined on some interval $\Omega \supset\left\{\omega_{1}, \omega_{2}, \ldots, \omega_{N}\right\}$. The problem can be presented as the minimization of the performance index

$$
I_{\Omega}=\sum_{k=1}^{N}\left\|c_{k}-F_{a}\left(\omega_{k}\right)\right\|_{\alpha_{k}},
$$

where $F_{a}\left(\omega_{k}\right)$ is a frequency characteristic of the approximation. The introduced weights $\alpha_{k} \geq 0$ can induce a filter effect of different values $c_{k}$ within interval $\Omega$. The employed measure $\|\cdot\|$ can be $L^{2}$ or another norm. In the case of a norm resulting in least-squares fitting, the minimization problem of $I_{\Omega}$ is easy to solve and the corresponding relations for the optimal solution can be easily derived. However, in the case of the matching problem, the application of the least-squares approach is not the best choice. On the other hand, the least sum of absolute errors ( $L S A)$ can be considered, but it induces poor convergence properties (Fiodorov, 1994; Janiszowski, 1998; Kozłowski, 2003). The nature of this effect can be explained by the following example.

Let us consider the problem of matching a position of a section of a known length, equal to 10 , by 6 measurements of the initial point $(I P)$ position

$$
\begin{aligned}
X_{1} & =\left\{x_{11}, x_{12}, \ldots, x_{16}\right\} \\
& =\{10.1,9.8,10.2,9.7,-10,10.3\}
\end{aligned}
$$

and 6 measurements of the end point $(E P)$ position

$$
\begin{aligned}
& X_{2}=\left\{x_{21}, x_{22}, \ldots, x_{26}\right\} \\
& \quad\{19.9,20.1,19.8,20.2,19.7,20.3\} .
\end{aligned}
$$

Let the performance index, used for the matching of the proper position of $I P$ and $E P$, be defined in the form of

$$
\begin{aligned}
I & =\sum_{k=1}^{6}\left\|I P-x_{1 k}\right\|^{\gamma}+\sum_{k=1}^{6}\left\|E P-x_{2 k}\right\|^{\gamma} \\
& =\sum_{k=1}^{6}\left\|I P-x_{1 k}\right\|^{\gamma}+\sum_{k=1}^{6}\left\|I P+10-x_{2 k}\right\|^{\gamma} \\
& =I(I P), \quad \gamma \geq 1 .
\end{aligned}
$$

Close inspection of the set $X_{1}$ shows one point $x_{15}=$ -10 quite distant from the other $x_{1 k}$. A good matching placement of the investigated section is the points $I P \approx$ 10 and $E P=10+I P \approx 20$, with the point $x_{15}$ neglected. This single outlier $x_{15}$ can be a result of some distortion, but an algorithm of matching should process this value, too.

Now let us consider the problem of matching the pair $(I P, E P)$ with different performance indices used. The Least-Squares (LS) matching corresponds to the value of $\gamma=2$ in (2). The most intuitive least sum of absolute errors is determined for $\gamma=1$. As the last option, will be investigated the performance index $L S E$ for a small exponent of $\gamma=1.1$.

In the case of the determination of LS matching $I P$ and $E P$ to the index (2), the solution will attain a minimal value of $I$ at $I P=8.749$ and $E P=18.749$. In the case of LSA value matching, the best fitting will yielded by the values $I P=9.9$ and $E P=19.9$ and will correspond to the above-mentioned intuitive solution. For LSE, the best matching will be produced by the solution $I P=9.872$ and $E P=19.872$, quite close to the $L S A$ solution, but not exactly the same. Now let us observe variations of $I(x)$ as functions of $x=I P,(E P=x+10)$ for different values of the parameter $\omega$, Fig. 1 . 


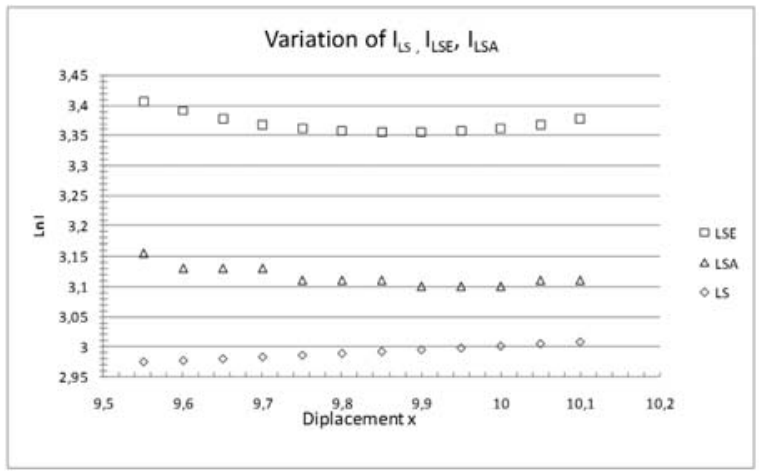

Fig. 1. Variations of performance indices $I_{L S}, I_{L S A}$ and $I_{L S E}$ in a logarithmic scale.

For the common presentation of trends of the compared indices, the values of $0.5 \cdot \ln \left(I_{L S}\right), \ln \left(I_{L S A}\right)$ and $\ln \left(I_{L S E}\right)$ in Fig. 1. The plots reflect some basic problems of matching with application of different methods, $L S$, $L S A$ and $L S E$. $L S$ matching is shifted quite apart from the intuitive solution $(I P=10, E P=20)$ and the index $I_{L S}$ slowly goes down and attains the minimal value for $I P=8.749$, but the corresponding algorithm for $I_{L S}$ minimization is very easy to implement. The most intuitive matching is attained by the LSA method, but it has a very serious drawback-the index value $I_{L S A}$ is constant within intervals of argument $x$, as shown in Fig. 1. This feature, reflecting the fact that the LSA algorithm can be performed only in an iterative way, represents problems that arise in numerical calculations (Fiodorov, 1994). The minimization of the LSA index yields intervals vanishing the numerical derivative. The plots, presented in Fig. 11 yield a conclusion that the best result for the $L S E$ algorithm is quite close to the $L S A$ matching, but the variation of $I_{L S E}$ is a continuous function of the argument $x$ with a unique minimum and therefore is more flexible in numerical processing.

The next section presents the formal statement of the approximation problem of fitting a transfer function to the known frequency characteristic. Derivation of the numerical approximation algorithm is based on reformulation of some nonlinear problem, resulting from matching complex values of the frequency characteristic for the investigated approximation $F_{a}$ to the pattern characteristic FR. The approximation problem is transformed to minimization of a measure of errors between patterns $\left|F R-F_{a}\right|$ defined by different performance indices- $L S, L S A$ or $L S E$. In Section 3 a simple theorem for the development of an effective way of calculation of solutions in the LSA and LSE sense is shown, followed by propositions of numerical algorithms for the approximation. The numerical calculations can be performed in a batch-processing scheme or can be based on the known, very old, recursive scheme, formerly developed by Young (1966), and next used in efficient on-line recursive algorithms, employed for parameter identification (e.g., Ljung and Söderström, 1987). The recursive version seems to be more flexible and easy launching numerical calculations. As shown in Section 4, the introduced approximation approach based on the pseudo-inverse scheme can be used for solving different tasks. For the verification and the efficiency test of the proposed approach, we present problems where the pattern is well known and the expected results are more or less intuitive: reduction of the model order for a discrete-time SISO process and approximation of the model in a continuous-time domain. The results of application of $L S E$ performance in the case of data with outliers compared with $L S$ results close the set of examples. The conclusions contain a summary of investigations and are focused on new applications of the proposed approach.

\section{Problem statement}

The set $F R=\left\{c_{k}, k=1 \ldots, N\right\}$ of the estimated discrete-time frequency response, defined on the set $\Omega=$ $\left\{\omega_{k}, k=1, \ldots, N\right\}$, represents dynamics of some SISO process. We are looking for a linear transfer function $G_{a}(s)$, of order $n$, that is defined by the relation

$$
G_{a}(s)=\frac{B(s)}{A^{\prime}(s)}=\frac{b_{0}+b_{1} s+\cdots+b_{n-1} s^{n-1}}{1+a_{1} s+\cdots+a_{n} s^{n}},
$$

where the coefficients $b_{i}, a_{i}$ are not known. The frequency characteristic for the approximation $G_{a}(s)$ will be determined as

$$
F_{a}\left(\omega_{k}\right)=\left.G_{a}(s)\right|_{s=l \omega_{k}}, \quad \omega_{k} \in \Omega
$$

or, in the case of investigating a process controlled by a zero-order sampling unit with the transfer function $I(s)$, by

$$
F_{a}\left(\omega_{k}\right)=\left.\left\{G_{a}(s) I(s)\right\}\right|_{s=j \omega_{k}}, \quad \omega_{k} \in \Omega .
$$

The measure of the approximation error is defined by the relation (1) with application of an index adjusted to the complex character of the frequency characteristic,

$$
\begin{aligned}
& I_{\alpha}^{\gamma}= \sum_{k=1}^{N} \alpha_{k}\left\{\left[\operatorname{Re} c_{k}-\operatorname{Re} F_{a}\left(\omega_{k}\right)\right]^{\omega}\right. \\
&\left.+\left[\operatorname{Im} c_{k}-\operatorname{Im} F_{a}\left(\omega_{k}\right)\right]^{\gamma}\right\} \\
&= \sum_{k=1}^{N}\left\|c_{k}-F_{a}\left(\omega_{k}\right)\right\|_{\alpha}^{\omega}, \\
& \omega_{k} \in \Omega, \quad \alpha_{k} \geq 0, \quad \gamma \in[1,2] .
\end{aligned}
$$

The approximation aim is to determine a set of coefficients $b_{i}, a_{i}$ (3), which attain a minimum of the performance index $I_{\alpha}^{\gamma}$. 
Let us denote by $\zeta_{k}$ the value of the frequency response of $G_{a}$ for $\omega_{k}$

$$
\begin{aligned}
G_{a}\left(j \omega_{k}\right) & =\left.\frac{b_{0}+b_{1} s_{k}+\cdots+b_{n-1} s_{k}^{n-1}}{1+a_{1} s_{k}+\cdots+a_{n} s_{k}^{n}}\right|_{s_{k}=j \omega_{k}} \\
& =\zeta_{k}, \quad k=1, \ldots, N
\end{aligned}
$$

The complex value $\zeta_{k}$ depends on polynomials $A$ and $B$ defined for complex frequency $s_{k}$,

$$
\begin{aligned}
& \zeta_{k}\left(1+A\left(s_{k}\right)\right)=B\left(s_{k}\right) \\
& \quad \Rightarrow \zeta_{k}=B\left(s_{k}\right)-\zeta_{k} A\left(s_{k}\right), \quad k=1, \ldots, N .
\end{aligned}
$$

The error $e_{k}=c_{k}-\zeta_{k} \in \mathbb{C}$ of the approximation is

$$
e_{k}=c_{k}-\zeta_{k}=c_{k}-B\left(s_{k}\right)+\zeta_{k} A\left(s_{k}\right)
$$

Now let us introduce some notation useful in the next steps of the algorithm:

$$
\begin{aligned}
B\left(s_{k}\right) & =\sum_{i=0}^{n-1} b_{i} s_{k}^{i}=B S_{k}, \\
B= & {\left[b_{0}, b_{1}, \ldots, b_{n-1}\right], } \\
S_{k}= & {\left[\begin{array}{c}
1 \\
s_{k} \\
\vdots \\
s_{k}^{n-1}
\end{array}\right], } \\
A\left(s_{k}\right)= & \sum_{i=1}^{n} a_{i} s_{k}^{i}=A s_{k} S_{k}, \\
A= & {\left[a_{1}, a_{2}, \ldots, a_{n}\right] } \\
A, B \in \mathbb{R}^{n}, & S_{k} \in \mathbb{C}^{n} .
\end{aligned}
$$

The unknown coefficients vectors $A, B$ are real, but the terms of the measure $I_{\alpha}^{\gamma}$ in (6) are in the complex domain. Let us distinguish the real and imaginary parts of $c_{k}$ and vectors representing the values of $B\left(s_{k}\right)$ and $\gamma_{k} A\left(s_{k}\right)$ in the relation (9) as

$$
\begin{gathered}
r_{k}=\operatorname{Re} c_{k}, \quad i_{k}=\operatorname{Im} c_{k} \\
R B_{k}=\left[\begin{array}{c}
1 \\
\operatorname{Re} s_{k} \\
\vdots \\
\operatorname{Re} s_{k}^{n-1}
\end{array}\right], \quad I B_{k}=\left[\begin{array}{c}
0 \\
\operatorname{Im} s_{k} \\
\vdots \\
\operatorname{Im} s_{k}^{n-1}
\end{array}\right], \\
R A_{k}=\left[\begin{array}{c}
\operatorname{Re}\left(s_{k} \zeta_{k}\right) \\
\operatorname{Re}\left(s_{k}^{2} \zeta_{k}\right) \\
\vdots \\
\operatorname{Re}\left(s_{k}^{n} \zeta_{k}\right)
\end{array}\right], \quad I A_{k}=\left[\begin{array}{c}
\operatorname{Im}\left(s_{k} \zeta_{k}\right) \\
\operatorname{Im}\left(s_{k}^{2} \zeta_{k}\right) \\
\vdots \\
\operatorname{Im}\left(s_{k}^{n} \zeta_{k}\right)
\end{array}\right] .
\end{gathered}
$$

The perfect fitting of the frequency characteristic of $G_{a}(s)$ to the pattern values $c_{k}$ will yield two conditions: $r_{k} \cong B R B_{k}-A R A_{k}, i_{k} \cong B I B_{k}-A I A_{k}$. The measure of the fitting error of the investigated approximation can be represented by the index

$$
\begin{aligned}
I_{\alpha}^{\gamma}(\Theta)= & \sum_{k=1}^{N} \alpha_{k}\left\{\left|r_{k}-B R B_{k}+A R A_{k}\right|^{\gamma}\right. \\
& \left.+\left|i_{k}-B I B_{k}+A I A_{k}\right|^{\gamma}\right\} \\
= & \sum_{k=1}^{N} \alpha_{k}\left\{\left|r_{k}-\Theta R_{k}\right|^{\gamma}+\left|i_{k}-\Theta I_{k}\right|^{\gamma}\right\}, \\
\Theta= & {[B, A] \in \mathbb{R}^{2 n}, } \\
R_{k}= & {\left[\begin{array}{c}
R B_{k} \\
-R A_{k}
\end{array}\right] \in \mathbb{R}^{2 n}, } \\
I_{k}= & {\left[\begin{array}{c}
I B_{k} \\
-I A_{k}
\end{array}\right] \in \mathbb{R}^{2 n} . }
\end{aligned}
$$

The minimization of the index $I_{\alpha}^{\gamma}(\Theta)$ for $N$ values of frequency $\omega$ involves the problem of minimization in $2 N$ points, due to the complex character of values $c_{k}$ and the form of the index (12). The algorithm for minimization depends on unknown values of $\zeta_{k}$ (9), which have to be estimated after determination of the model coefficients vector $\Theta$. Hence, the minimization algorithm has to be arranged in an iterative way.

The minimization of

$$
\begin{gathered}
I_{\alpha}^{\gamma}(\Theta)=\|V \Theta-Y\|_{\alpha}^{\gamma} \rightarrow \min _{\Theta}, \\
V=\left[\begin{array}{c}
R_{1} \\
I_{1} \\
\vdots \\
R_{N} \\
I_{N}
\end{array}\right], \quad Y=\left[\begin{array}{c}
r_{1} \\
i_{1} \\
\vdots \\
r_{N} \\
i_{N}
\end{array}\right], \\
\alpha=\operatorname{diag}\left[\alpha_{1}, \alpha_{2}, \ldots, \alpha_{N}\right], \quad R_{i}, I_{i} \in \mathbb{R}^{2 n},
\end{gathered}
$$

can be arranged in different ways. In the paper it is assumed that the resulting transfer function coefficient will yield a frequency response close to the known values of $c_{k}$, and therefore in the first step the values $\zeta_{k}$ were replaced by $c_{k}$ in (11). The next runs of the algorithm have to be iterative, with determination of $\zeta_{k}$ calculated as in (7). A batch-processing algorithm is proposed as Algorithm 1.

The start of a minimization algorithm for $I_{\alpha}^{2}\left(\Theta_{0}\right)$ is simple. The $L S$ approximation is direct and after basic calculations can be solved as shown in (14), where $j=0$ is the index of the iteration

$$
\begin{gathered}
\Theta_{j}^{L S}=\left[V^{j^{T}} \alpha V^{j}\right]^{-1} V^{j^{T}} \alpha Y, \quad \alpha \in \mathbb{R}^{N \times N}, \\
V^{j}=\left[\begin{array}{c}
R_{1}^{j} \\
I_{1}^{j} \\
\vdots \\
R_{N}^{j} \\
I_{N}^{j}
\end{array}\right],
\end{gathered}
$$


Algorithm 1. Batch-processing.

Step 0. At the start of iterations, the initial guess of $\zeta_{k}^{0}$ is substituted by $c_{k}, k=1, \ldots, N$. For the known frequency values $\omega_{k}$ the vectors $R B_{k}, I B_{k}, R A_{k}, I A_{k}$ in (11) can be calculated and the estimate $\Theta_{0}$ can be determined by minimization of $I(\Theta)$ in (13).

Step 1. For the known initial component vector $\Theta_{0}$ the values of $\zeta_{k}^{1}, k=1, \ldots, N$ can be recalculated, consistent with the determination (7), (11) of the vectors $R B_{k}, I B_{k}, R A_{k}, I A_{k}$. The next step of minimization (13) can be performed and yields the estimation of the vector of the model coefficients $\Theta_{1}$. After this step, based on (7), the estimate of the model error and the resulting performance index $I_{\alpha}^{\gamma}\left(\Theta_{1}\right)$ in (12) are calculated.

Step 2 and onward. For the known vector $\Theta_{j}$ the values of $\zeta_{k}^{j}, k=1, \ldots, N$ can be calculated and the next iteration of the vectors $R B_{k}, I B_{k}, R A_{k}, I A_{k}$ is performed. The next step of minimization of (13) $I_{\alpha}^{\gamma}\left(\Theta_{j}\right)$ is made and yields the next approximation of $\Theta_{j+1}$. The minimization of (13) proceeds until the value of $I_{j}$ is decreasing.

The only problem here is the convergence of the proposed algorithm. Step 0 always produces an estimate $\Theta_{0}$, which can be close to or quite distant from the pattern set $F R$. The process of minimization of $I^{2}$ has to be supervised. After the first 2 to 3 steady iterations of this algorithm, the procedure is convergent. However, sometimes, especially in the case of quite a small order $n$ of the transfer function $G_{a}(s)$ in (7), the algorithm may converge to a false solution. In the presence of big outliers in the pattern plot of $c_{k}$, this effect was observed, too.

Now we will discuss algorithms for effective calculation with non-square performance indices, with exponent $\gamma<2$. These algorithms are not based on direct rules as in (14) and need a recursive way of calculations.

\section{Minimization of LSA and LSE error measures}

Let us first consider the well-known problem of minimization in the sense of the weighted minimal sum of error squares of the linear regression model

$$
\hat{Y}=V \Theta, \quad \hat{Y} \in \mathbb{R}^{M}, \quad V \in \mathbb{R}^{M \times m}, \quad \Theta \in \mathbb{R}^{m}
$$

for the vector $Y \in \mathbb{R}^{M}$, where $V$ is a matrix of stored data and $\Theta$ is a vector of unknown model coefficients. The least-squares estimation for the minimization of the model error in the weighted $L S$ sense (Ljung and Söderström,
1987),

$$
\begin{gathered}
I_{W L S}=\left\{(Y-V \Theta)^{T} W(Y-V \Theta)\right\} \\
W \in \mathbb{R}^{M \cdot M}, \\
\operatorname{diag}\left[w_{1}, \ldots, w_{M}\right], \quad w_{i} \geq 0,
\end{gathered}
$$

where $W$ is the matrix of weights, is defined by the well-known formula (Ljung and Söderström, 1987)

$$
\Theta_{W L S}=\left[V^{T} W V\right]^{-1} V^{T} W Y .
$$

Now let us consider a special form of the WLS estimator with dynamic weight coefficients $w$, dependent on the error volume produced by the resulting model.

Theorem 1. Consider a linear form

$$
\hat{Z}=X \psi, \quad \hat{Z} \in \mathbb{R}^{M}, \quad X \in \mathbb{R}^{M \times m}, \quad \psi \in \mathbb{R}^{m},
$$

which is a model of a variable $z$, presented by the vector $Z=\left[z_{1}, z_{2}, \ldots, z_{M}\right]^{T}$ and $X$ is a matrix composed of row vectors $x_{k} \in \mathbb{R}^{m}, k=1, \ldots, M$. If $\psi^{\prime \prime} \in \mathbb{R}^{m}$ denotes a vector of the model coefficients, which attains a minimum of the performance index

$$
I=\sum_{k=1}^{M}\left\|z_{k}-x_{k} \psi^{\prime \prime}\right\|^{\gamma}, \quad x_{k} \in \mathbb{R}^{m}, \quad \gamma \geq 1,
$$

and $E$ is equal to

$$
\begin{gathered}
E=\operatorname{diag}\left\{e_{1}, e_{2}, \ldots, e_{M}\right\}, \\
e_{k}=\frac{1}{\left\|z_{k}-\hat{z_{k}}(\psi)\right\|^{2-\gamma}}, \quad k=1, \ldots, M, \\
\hat{z}_{k}=x_{k} \psi^{\prime \prime},
\end{gathered}
$$

then the vector $\psi^{\prime \prime}$ satisfies the relation

$$
\psi^{\prime \prime}=\left[X^{T} E X\right]^{-1} X^{T} E Z .
$$

Proof. The sum $I\left(\psi^{\prime \prime}\right)=\sum_{k=1}^{M}\left|z_{k}-\hat{z}_{k}(\psi)\right|^{\gamma}$ can be presented as

$$
I\left(\psi^{\prime \prime}\right)=\sum_{k=1}^{M}\left[\left(z_{k}-\hat{z}_{k}(\psi)\right)^{2}\right]^{\gamma / 2} .
$$

$I$ is a convex function of $\psi$. A necessary condition for a minimum of $I(\psi)$ yields

$$
\begin{aligned}
& \frac{\partial}{\partial \psi} I(\psi) \\
& =\frac{\partial}{\partial \psi} \sum_{k=1}^{M}\left[\left(z_{k}-\hat{z}_{k}(\psi)\right)^{2}\right]^{\gamma / 2} \\
& =\sum_{k=1}^{M} \frac{\gamma}{2\left[\left(z_{k}-\hat{z}_{k}(\psi)\right)^{2}\right]^{(2-\gamma) / 2}} \frac{\partial}{\partial \psi}\left(z_{k}-\hat{z}_{k}(\psi)\right)^{2}
\end{aligned}
$$$$
\equiv 0 \text {. }
$$ 
This relation can be presented in the following form:

$$
\begin{aligned}
\frac{\partial}{\partial \psi} I & =\sum_{k=1}^{M} e_{k} \frac{\partial}{\partial \psi}\left(z_{k}-\hat{z}_{k}(\psi)\right)^{2} \equiv 0, \\
e_{k} & =\frac{1}{\left[\left(z_{k}-\hat{z}_{k}(\psi)\right)^{2}\right]^{(2-\gamma) / 2}} .
\end{aligned}
$$

The above expression is equivalent to the necessary condition for a minimum of the weighted least square problem (e.g., Ljung and Söderström, 1987), where the weights are equal to $e_{k}=1 / \epsilon_{k}$, and then the coefficient vector $\psi^{\prime \prime}$ is determined as in (21). This completes the proof.

The theorem yields the condition for $\psi^{\prime \prime}$, but first the vector of estimation errors $e=\left[e_{1}, e_{2}, \ldots, e_{n}\right]$ has to be known. In case of off-line estimation, Algorithm 2 is proposed.

$\overline{\text { Algorithm 2. Off-line estimation in the LSE sense. }}$ $e_{j}=1, j=1, \ldots, M$,

$$
\psi^{0}=\left[X^{T} X\right]^{-1} X^{T} Z .
$$

Step 2. Determine the absolute values of output errors $\epsilon_{k}^{0}$,

$$
\begin{gathered}
\epsilon_{k}^{0}=\left|z_{k}-\hat{z}_{k}(\psi)\right|^{\gamma}, \quad k=1, \ldots, M, \\
\gamma \in[1,1.2],
\end{gathered}
$$

and set the weighing coefficients

$$
\begin{gathered}
e_{k}^{0}= \begin{cases}1 / \epsilon_{k}, & \epsilon_{k}>\epsilon_{\min }, \\
\epsilon_{k}=\max , & \epsilon_{k} \leq \epsilon_{\min },\end{cases} \\
k=1, \ldots, M, \quad \max =1 / \epsilon_{\min }
\end{gathered}
$$

where $\epsilon_{\min }$ can be determined by quantization or measurement errors. For the initial step $j=0$ the weight matrix is equal to

$$
E^{0}=\operatorname{diag}\left[e_{1}^{0}, e_{2}^{0}, \ldots, e_{M}^{0}\right] .
$$

Step 3. Compute the estimate $\psi^{1}$ in 21 ,

$$
\psi^{1}=\left[X^{T} E^{0} X\right]^{-1} X^{T} E^{0} Z,
$$

with weights $e_{k}^{1}$ defined as in (24).

Step 4. For the next iterations with indices $j=2,3, \ldots$

Steps 2 and 3 are repeated with new determined model errors 23 and updated values in the weight matrix $E^{j}$ (25) until the index $I$ in 19 reaches a limit value, i.e., it is not decreasing within a determined number $\kappa$ of the last runs.
Algorithm 2 is based on batch processing of recorded data. Due to the observed behaviour of the LSA performance index presented in the Introduction, the convergence of this iterative processing may yield some problems (Fiodorov, 1994; Janiszowski, 1998). LSE estimation yields better convergence properties. The only difference between $L S A$ and $L S E$ estimation is the value of the weights coefficients (20), and the $L S E$ index can be expressed as

$$
\begin{aligned}
I & =\sum_{k=1}^{M}\left|z_{k}-x_{k} \psi_{L S E}\right|^{\gamma} \\
& =\sum_{k=1}^{M} e_{k}\left|z_{k}-x_{k} \psi_{L S E}\right|^{2}, \\
e_{k} & =\left|z_{k}-x_{k} \psi_{L S E}\right|^{\lambda}, \quad \lambda=2-\gamma,
\end{aligned}
$$

and in effect these weights $e_{k}$ will be used in estimation (26) for $L S E$ off-line estimation.

The batch processing algorithm yields one problem in calculations: the first estimates in LSA or LSE are received after the processing of all $M$ recorded points.

The convergence rate can be improved when estimation for one run, i.e., $M$ steps, is determined recursively, like in on-line schemes (Janiszowski, 1998; Kozłowski, 2003; Kowalczuk and Kozłowski, 2011). The efficiency of the recursive scheme was observed quite long ago (Young, 1966), and next its positive features were confirmed (Ljung and Söderström, 1987) and used in other applications (Janiszowski, 1998; Kozłowski, 2003; Kowalczuk and Kozłowski, 2011). A proposition of such an algorithm is shown below.

In comparison with the usually used on-line identification schemes, where the index $k$ represents discrete time instants, in the above algorithm this index represents values of radial frequency $\omega_{k} \in \Omega$. The choice of these values is discussed in the next section. The proposed starting point 35 can be used or a primary fitting with the $L S$ scheme for $\lambda=2$ can be tested.

The application of the recursive calculation 28) - 35 to estimation of model coefficients has an advantage with respect to the batch processing scheme proposed by (22) - 26). From the very beginning the approximation $\Theta$ slowly but steadily converges to the expected $L S E$ or $L S A$ estimates, does not contain an initial phase of the $\psi_{L S A}^{0}$ estimation 22, which can push the initial result far away from the proper resolution. This advantage is accompanied by a convergence problem. The first iterations of $\psi_{L S E}(j)$ have to be well prepared, i.e., the values of $\omega_{1}, \ldots, \omega_{q},(q \ll M)$, close to 0 , yield very small variations in $c_{k}$ and the convergence of estimation $\psi_{L S E}(j)$ in this phase of data processing is slow and not very well conditioned. Therefore, a set of the values $c_{k}$ has to be carefully selected to yield sufficient changes into 
Algorithm 3. On-line estimation in the LSA sense.

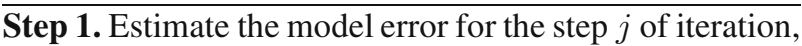

$$
\begin{gathered}
\epsilon_{k}=z_{k}-x_{k} \psi_{L S E}(k-1), \quad k=1, \ldots, M, \\
x_{k}=\left[x_{1 k}, x_{2 k}, \ldots, x_{m k}\right] .
\end{gathered}
$$

Step 2. Determine the weight coefficient $e_{j}$,

$$
e_{k}= \begin{cases}1 /\left|\epsilon_{k}\right|^{\lambda}, & \left|\epsilon_{k}\right| \geq \epsilon_{\min } \\ \max =1 / \epsilon_{\min }, & \left|\epsilon_{k}\right|<\epsilon_{\min }\end{cases}
$$

Step 3. Calculate the vector of the filtered model inputs and gain vector $\chi$,

$$
\begin{gathered}
v_{k}=e_{k} x_{k}, \quad v_{k} \in \mathbb{R}^{m}, \\
\mu_{k}=\left[\rho+v_{k} \mathbf{P}_{k-1} v_{k}^{T}\right]^{-1}, \quad \mu_{k}, \rho \in \mathbb{R}, \\
\chi=\mu_{k} \mathbf{P}_{k-1} v_{k}^{T}, \quad \chi \in \mathbb{R}^{m} .
\end{gathered}
$$

Step 4. Update the model coefficient vector,

$$
\begin{gathered}
\psi_{L S E}(k)=\psi_{L S E}(k-1)+\operatorname{sign}\left(\epsilon_{k}\right) \chi\left|\epsilon_{k}\right|^{\gamma-1}, \\
\alpha_{L S E} \in \mathbb{R}^{m} .
\end{gathered}
$$

Step 5. Update the estimate of the matrix $\mathbf{P}$,

$$
\mathbf{P}_{k}=\left[1-\chi v_{k}\right] \mathbf{P}_{k-1} \rho^{-1}, \quad \mathbf{P}_{k} \in \mathbb{R}^{m m},
$$

where $\rho=[0.99,1.0]$ is a forgetting factor.

Step 6. The initial conditions for the above algorithm are

$$
\begin{gathered}
\psi_{L S E}(0)=[0,0, \ldots, 0], \quad \alpha_{L S E} \in \mathbb{R}^{m}, \\
\mathbf{P}_{0}=\operatorname{diag}(\kappa),
\end{gathered}
$$

and $\kappa \in\left[10^{8}, 10^{20}\right]$ initialises the matrix $(P)$.

estimation of $c_{k}$ in the approximation process. This topic will be investigated and considered in further sections.

\section{Application of the proposed algorithm for approximation and reduction of the model order for SISO systems}

The problem of approximation can have many applications like, e.g., reduction of the order of discrete-time model dynamics for SISO system, approximation of the process model in a continuous time domain based on the discrete-time frequency response with reduction of the model order (or without), approximation of a model in a discrete or a continuous time domain with the filtering of the response in a specified frequency interval, change in the sampling interval $\Delta$ in discrete-time representation and others. In all these applications the main tool is efficient approximation in the frequency domain, and the final result can be achieved by an adequate statement of the problem and proper pre-processing of the data used.

The proposed approach will be first tested for reduction of the model order of a dynamic process. In this application the expected result is usually quite obvious and the efficiency of the approximation algorithm can be verified. The quality of approximation has to be verified, and therefore the following normalized performance index is proposed:

$$
I_{n}=\left\{\frac{1}{M} \sum_{k=1}^{M} \frac{\left|c_{k}-G\left(j \omega_{k}\right)\right|^{2}}{\left|c_{k}\right|^{2}}\right\}^{1 / 2} .
$$

This form of the performance index does not impose any frequency a priori due to the introduced weighing normalizing factor $\left|c_{k}\right|^{2}$ in the denominator. Its form is different from the suggested $L S A$ or $L S E$ form (6) but corresponds to indices used in the literature (Ljung and Söderström, 1987; Mohan et al., 2004; Sreeram and Agatokhlis, 1991) and therefore can be used for comparison.

4.1. Reduction of the model order for a discretetime linear model of a SISO process. This problem usually is yielded as a result of process identification, performed with defined sampling interval $\Delta$. The estimated discrete-time transfer function of the SISO process,

$$
\begin{aligned}
G^{*}\left(z^{-1}\right) & =\frac{B\left(z^{-1}\right) z^{-d}}{1+A\left(z^{-1}\right)} \\
& =\frac{\left(b_{0}+b_{1} z^{-1}+\cdots+b_{p}\right) z^{-d}}{1+a_{1} z^{-1}+\cdots+a_{p} z^{-p}}, \\
T_{0} & =d \Delta,
\end{aligned}
$$

with the specified delay $T_{0}$ is next investigated in the form of the lower order $r \leq p$ model (37) with the same discrete-time delay $d$. At the beginning a set $F R$ of given frequency characteristic values has to be created for some set $\Omega$. The set of frequency values $\Omega$ has an upper bound $\omega_{\text {up }}$, in the given case limited by the Shannon's frequency $\pi / \delta$. The initial frequency $\omega_{1}=\omega_{\text {low }}$ and the following values $\omega_{k}$, its number and choice is a decision to be made by the user. In our case, the value of $\omega_{1}$ is set to $10^{-5} \cdot \omega_{\text {up }}$. The number of generated frequency values $\omega_{k}$ should be sufficiently large and is defined, in the presented examples, as $M^{\prime} \gg 300$. The interval $\left[\omega_{\text {low }}, \omega_{\text {up }}\right]$ is first divided in a logarithmic scale according to the rule

$$
\Delta \omega=\exp \left\{\frac{\ln \left(\omega_{\text {up }} / \omega_{\text {low }}\right)}{M^{\prime}}\right\}, \quad \omega_{1}=\omega_{\text {low }} .
$$


Next, the following selection of the frequency $\omega$ value is introduced. The value $\omega_{k} \in \Omega$ is multiplied by $\Delta \omega$. If the new value $\omega^{\prime}=\omega_{k} \cdot \Delta \omega$ presents a sufficient variation of argument, i.e., $\arg G^{*}\left[j\left(\omega^{\prime}\right)\right]-$ $\arg G^{*}\left[j\left(\omega_{k}\right)\right]>\Delta \arg$, then this value $\omega^{\prime}$ is included into $\Omega$ as $\omega_{k+1}$. Otherwise, $\omega^{\prime}$ is increased again, $\omega^{\prime}=$ $\omega^{\prime} \cdot \Delta \omega$ and the condition for argument variation is tested. The above rule generates a set $\Omega$ of $M$ frequency values $\omega_{k}$ that preserve a sufficient variation of the investigated frequency response,

$$
\begin{gathered}
\Omega=\left\{\omega_{k}, k=1, \ldots, M\right\} \\
F R=\left\{c_{k}=G^{*}\left(j \omega_{k}\right), k=1, \ldots, M\right\}, \\
\left|\arg G^{*}\left(j \omega_{k}\right)-\arg G^{*}\left(j \omega_{k-1}\right)\right|>\Delta \arg , \\
k=1, \ldots, M .
\end{gathered}
$$

As an example, we use used a model of the eight order discrete-time transfer function (Sreeram and Agatokhlis, 1991),

$$
G_{8}^{*}=\frac{a_{8}(z-1)}{b_{8}(z-1)}
$$

where

$$
\begin{aligned}
a_{8}(z-1)= & 0.2012 z^{-1}+0.1395 z^{-2}-0.0263 z^{-3} \\
& +0.0190 z^{-4}-0.0645 z^{-5}-0.0328 z^{-6} \\
& +0.0055 z^{-7}-0.0088 z^{-8} \\
b_{8}(z-1)= & -0.6307 z^{-1}+0.4185 z^{-2}-0.0788 z^{-3} \\
& +0.0570 z^{-4}+0.1935 z^{-5}+0.0983 z^{-6} \\
& -0.0165 z^{-7}+0.0023 z^{-8} .
\end{aligned}
$$

In the work of Sreeram and Agatokhlis (1991) the reduction into a second order model yields the following transfer function:

$$
G_{2}^{*}\left(z^{-1}\right)=\frac{0.23159 z^{-1}-0.15233 z^{-2}}{1-1.75851 z^{-1}+0.83862 z^{-2}},
$$

which, after evaluation with $I_{n}$ in (36), gives the value of $I_{n}=0.043$. The application of the proposed $L S E$ approach, with $\gamma=1.2$, based on $M=289$ and points selected as in (38), (39) gives the following results for reduction to the third and second orders:

$$
\begin{aligned}
& G_{3}^{L S E}\left(z^{-1}\right) \\
& =\frac{0.21309 z^{-1}-0.21427 z^{-2}+0.04096 z^{-3}}{1-2.21016 z^{-1}+1.63034 z^{-2}-0.37974 z^{-3}}
\end{aligned}
$$

and the performance index $I_{n}$ equals $6.1 \cdot 10^{-5}$, which is a very good result. The result for second order reduction is quite good, too,

$$
G_{2}^{L S E}\left(z^{-1}\right)=\frac{0.23089 z^{-1}-0.15083 z^{-2}}{1-1.75752 z^{-1}+0.83751 z^{-2}},
$$

and is qualified with $I_{n}=0.0047$. Both results present quite good fitting and show that the LSE estimation scheme can be satisfactorily efficient.

4.2. Approximation in the continuous time domain, based on frequency characteristics derived from a discrete-time model of a SISO process. This problem usually appears during process identification based on sampled data, when the determined model has to be expressed in continuous time form, which is more easy to handle and evaluate. It is clear that sampled data deliver information in a limited frequency domain $\left[0, \omega_{S}\right]$. Quite often data are gathered in an experiment where the investigated process is controlled by a microcontroller device, with constant sampling time $\Delta$. This fact has to be included into determination of investigated dynamics. The observed dynamic properties in the form of frequency characteristic, denoted by $c_{k}^{\prime}$, are products of process and sampling unit $G_{s}$ dynamics

$$
\begin{aligned}
c_{k}^{\prime} & =G^{*}\left(j \omega_{k}\right) \cdot G_{s}\left(j \omega_{k}\right), \\
G_{s}\left(j \omega_{k}\right) & =\frac{1-\exp \left(-j \omega_{k} \Delta\right)}{j \omega_{k}} .
\end{aligned}
$$

The determined values of the frequency characteristic have to be redefined at the beginning of the approximation process,

$$
c_{k}=c^{\prime}\left[G_{s}\left(j \omega_{k}\right)\right]^{-1}, \quad k=1, \ldots, M .
$$

These transformed values $c_{k}$ are next used for approximation of the transfer function for investigated continuous time representation of a SISO process.

The efficiency of the proposed approach can be presented on an example of a simple, second order linear dynamic process, described by a transfer function of the form

$$
G_{2}(s)=\frac{20}{1+40 s}-\frac{1}{1+4 s}=\frac{19+40 s}{1+44 s+160 s^{2}} .
$$

This transfer function was used for generation of a set of data with sampling interval $\Delta=1 \mathrm{~s}$. In this example the sensitivity of approximations to the presence of outliers is tested. The process of gathering frequency characteristic data was performed

1. without any distortions $(F C)$,

2. with outliers added to the initial set $F C$ with more points of reduced values $\left(F C_{-}\right)$,

3. with outliers added to the initial set $F C$ with more points of increased values $\left(F C_{+}\right)$.

All data sets $F C, F C_{-}, F C_{+}$, containing each 300 points, are generated with an algorithm satisfying conditions presented in relation (39). Next, these data 
are used for approximation of the resulting transfer function. The outliers are introduced by changing the corresponding amplitudes of $c_{k}$ and observing the reaction of approximation algorithm for such distortions.

In the case of undisturbed data $F C$ and the second order of the investigated transfer function, the $L S, L S E$ and LSA approximation results are practically the same and equal to the original transfer function $G_{2}(s)$ (45). But for the first order approximations for the $L S, L S A$ and $L S E$ schemes, the results are different:

$$
\begin{aligned}
G_{1 L S}(s) & =\frac{19.38452}{1+48.30591 s}, \\
G_{1 L S A}(s) & =\frac{19.00002}{1+41.89483 s}, \\
G_{1 L S E}(s) & =\frac{19.00003}{1+41.89572 s} .
\end{aligned}
$$

The $L S$ approximation is visibly worse, which can be observed in Fig. 2, where the plots for the $L S$ and $L S A$ approximation, together with the original frequency characteristic $F C$, are shown. We can observe a difference at low frequency values $\omega_{k}$ : the gain of the $L S$ approximation is too high. This effect is visible considering the form of the transfer function $G_{1 L S}(s)$, too. The static gain for this transfer function is equal to 19.384. The frequency characteristic begins from 19 and quite the same value is present in approximations $G_{1 L S E}(s)$ and $G_{1 L S A}(s)$. The differences between these transfer functions were negligible and therefore only the frequency characteristic for $G_{1 L S A}(s)$ is presented in Fig. 2] At the high frequencies $\omega_{k}$, both approximations $L S$ and $L S A$ present the same plot, typical for the first order transfer function.

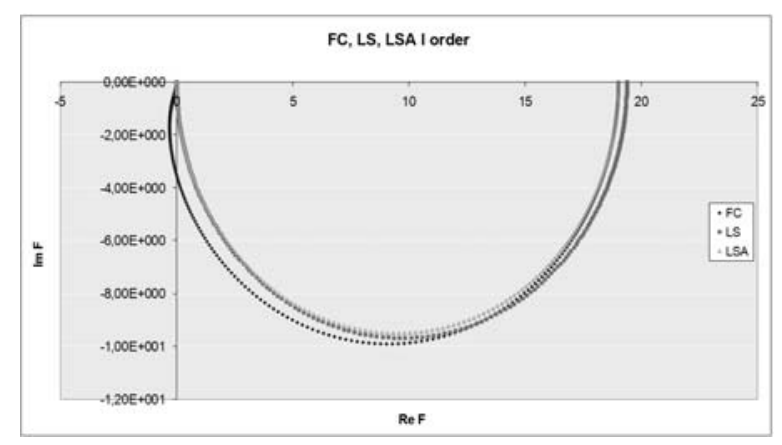

Fig. 2. Frequency characteristics for the first-order approximations determined with the $L S$ and $L S A$ schemes compared with the original pattern $F C$.

The observed difference is not the only one. In the case of outliers in the frequency pattern $F C_{-}$, can be observed, cf. some other behaviour of the approximations, Fig. 3. The set $F C_{-}$of frequency characteristics, with visible outliers, is used for the determination of the first order approximations with the $L S E$ and $L S$ algorithms.
In this case, the $L S E$ approximation scheme produces quite the same effect as in the case of the original set $F C$. The outliers visibly destroy the variation of the $L S_{-}$ approximation plot. The attraction of outliers pushed the resulting $L S_{-}$approximation quite apart from the set $F C_{-}$.

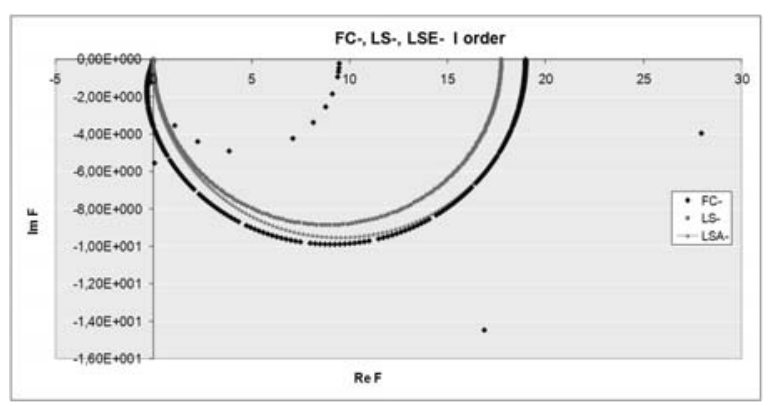

Fig. 3. Frequency characteristics for the first-order approximations $L S_{-}$, the $L S A_{-}$determined with $L S A_{-}$and $L S$ schemes, compared with the pattern $F C_{-}$with outliers.

The case of positive gathered outliers is presented in Fig. 4 The approximations $L S_{+}$and $L S E_{+}$ represent approximation results with application of the corresponding algorithms, but this time for the second order of the transfer function. The difference between $L S E_{+}$and $F C$ is hardly visible with distinctions of outliers. The $L S_{+}$results are different-again the outliers pushed the $L S_{+}$results in the direction of the transfer with an increased gain.

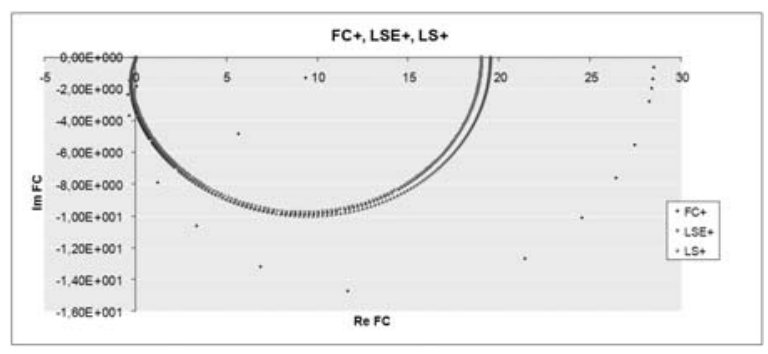

Fig. 4. Frequency characteristics for the second-order approximations $L S_{-}, L S E_{-}$determined with the $L S E$ and $L S$ schemes, compared with the pattern $F C_{-}$with outliers.

The transfer functions determined within approximation processing are equal to

$$
\begin{aligned}
G_{2 L S+}(s) & =\frac{19.49801+29.6896 s}{1+44.63748 s+118.786 s^{2}}, \\
G_{2 L S E+}(s) & =\frac{19.03859+28.5043 s}{1+44.6002 s+114.020 s^{2}} .
\end{aligned}
$$

Brief inspection of these forms confirms an increased static gain in the $L S_{+}$approximation and practically the proper value in the $L S E_{+}$approximation result. The index 
(36) was not calculated because of the presence of the outliers. However, comparison of the presented figures shows that the $L S E_{+}$approximation covers practically the original plot $F C_{+}$excluding the outliers. $L S_{+}$is close but reflects a small displacement in the direction of the outliers.

\section{Conclusions}

The approach presented in this paper is based on the approximation algorithm with the option of minimization of the non-quadratic error measure. The results shown confirm that both aspects can be combined in one algorithm and deliver good results. The primary iterative working batch processing algorithm (7) 13 considered presents worse convergence than the recursive version (26)-(34). This effect can be a result of recursive scheme flexibility. The solution is in fact improved in each step of the recursive processing. Interesting is the fact that the recursive algorithm, invented initially for statistic estimation of dynamic models using measured time series, ca. 50 years ago (Young, 1966), can be now successfully used for calculation of some other problems, like recursive approximation. However, it should be stressed that positive results can be obtained only after special processing (38) -39) of data. This choice of the frequency set $\Omega$ and the set of the corresponding complex values $F R$ (39) can be completed by a stochastic draw of pairs $\left\{\omega_{k}, c_{k}\right\}$ for the recursive algorithm (28)- This procedure does not present unstable solutions mentioned in Section 3. Future works on the proposed algorithm will consider application in the case of weakly nonlinear continuous time systems, e.g., a chemical reactor with dynamics dependent on operation point.

Recursive fitting, based on the $\operatorname{LSE}(\gamma=1.2)$ performance index $I_{\Omega}^{\gamma}$ is quite effective and very close to $L S A$ approximation. This observation, shown in Example 2, was confirmed for different investigated cases. In all cases the $L S E$ recursive approximation procedure was stable and produced reasonable results. Introduction of a variable value of the exponent $\gamma$ into the performance index created an additional degree of freedom into algorithms, based on minimization of performance indices as introduced in (1) or (12).

The proposed approach can create a new way for solving problems where non-quadratic criteria are reasonable and shall be used, especially where outliers create problems of $L S$ approach convergence.

\section{Acknowledgment}

The paper was partially supported by a grant of the Dean of the Mechatronic Department of the Warsaw University of Technology for 2012.

\section{References}

Deschrijver, D., Gustavsen, B. and Dhaene, T. (2007). Advancements in iterative methods for rational approximation in the frequency domain, IEEE Transactions on Power Delivery 22(3): 1633-1642.

Deschrijver, D., Knockaert, L. and Dhaene, T. (2010). Improving robustness of vector fitting to outliers in data, IEEE Electronics Letters 46(17): 1200-1201.

Deschrijver, D., Knockaert, L. and Dhaene, T. (2011). Robust macromodeling of frequency responses with outliers, $15 \mathrm{th}$ IEEE Workshop on Signal Propagation on Interconnects (SPI), Naples, Italy, pp. 21-24.

Fiodorov, E. (1994). Least absolute values estimation: Computational aspects, IEEE Transactions on Automatic Control 39(3): 626-630.

Grivet-Talocia, S., Bandinu, M. and Canavero, F. (2005). An automatic algorithm for equivalent circuit extraction from noisy frequency responses, 2005 International Symposium on Electromagnetic Compatibility, EMC 2005, Zurich, Switzerland, Vol. 1, pp. 163-168.

Gustavsen, B. (2004). Wide band modeling of power transformers, IEEE Transactions on Power Delivery 19(1): 414-422.

Gustavsen, B. (2006). Relaxed vector fitting algorithm for rational approximation of frequency domain responses, $I E$ EE Workshop on Signal Propagation on Interconnects, Berlin, Germany, pp. 97-100.

Gustavsen, B. and Mo, O. (2007). Interfacing convolution based linear models to an electromagnetic transients program, Conference on Power Systems Transients, Lyon, France, pp. 4-7.

Gustavsen, B. and Semlyen, A. (1999). Rational approximation of frequency domain responses by vector fitting, IEEE Transactions on Power Delivery 14(3): 1052-1061.

Janiszowski, K. (1998). Towards least sum of absolute errors estimation, IFAC Symposium on Large Scale Systems LSS'98, Patras, Greece, pp. 613-619.

Kowalczuk, Z. and Kozłowski, J. (2011). Non-quadratic quality criteria in parameter estimation of continuous-time models, IET Control Theory \& Applications 5(13): 1494-1508.

Kozłowski, J. (2003). Nonquadratic quality indices in estimation, approximation and control, IEEE Conference MMAR'2003, Międzyzdroje, Poland, pp. 277-282.

Levy, E.C. (1959). Complex-curve fitting, IRE Transactions on Automatic Control AC-4(1): 37-43.

Lima, A.C.S., Fernandes, A. and Carneiro, S., J. (2005). Rational approximation of frequency domain responses in the $\mathrm{s}$ and $\mathrm{z}$ planes, IEEE Power Engineering Society General Meeting, San Francisco, CA, USA, Vol. 1, pp. 126-131

Ljung, L. and Söderström, T. (1987). Theory and Practice of Recursive Identification, MIT Press, Cambridge, MA.

Mohan, R., Choi, M.J., Mick, S., Hart, F., Chandrasekar, K., Cangellaris, A., Franzon, P. and Steer, M. (2004). 
Causal reduced-order modeling of distributed structures in a transient circuit simulator, IEEE Transactions on Microwave Theory and Techniques 52(9): 2207-2214.

Pintelon, R. and Schoukens, J. (2004). System Identification: A Frequency Domain Approach, John Wiley \& Sons, New York, NY.

Sreeram, V. and Agatokhlis, P. (1991). Model reduction of linear discrete-time systems via impulse response Gramians, International Journal on Control 53(1): 129-144.

Unbehauen, H. and Rao, G. (1997). Identification of continuous-time systems: A tutorial, 11th IFAC Symposium on System Identification, Kitakyushu, Japan, pp. 1023-1049.

Varricchio, S., Gomes, S. and Martins, N. (2004). Modal analysis of industrial system harmonics using the s-domain approach, IEEE Transactions on Power Delivery 19(3): 1232-1237.
Wahlberg, B. and Mäkilä, P. (1996). On approximation of stable linear dynamical systems using Laguerre and Kautz functions, Automatica 32(5): 693-708.

Young, P.C. (1966). Process parameter estimation and self adaptive control, in P.H. Hammnod (Ed.), Theory of Self Adaptive Control Systems, Vol. 1, Plenum Press, New York, NY, p. 118.

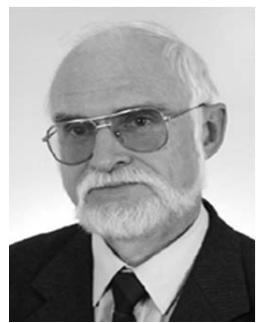

Krzysztof B. Janiszowski, a leader of the Activators for Automation and Robotics Division at the Mechatronics Department of the Warsaw University of Technology, specializes in identification, modeling and control techniques. His application areas include identification of industrial plants, algorithms for control and fast prototyping in fluidic servo-mechanisms, models of human circulation systems, software packages for modeling and control of industrial processes.

Received: 7 December 2012

Revised: 5 July 2013

Re-revised: 12 October 2013 